NASA Technical Memorandum 107011

AIAA-95-1210

\title{
Micromechanics of Composites With Shape Memory Alloy Fibers in Uniform Thermal Fields
}

Victor Birman

University of Missouri-Rolla

St. Louis, Missouri

Dimitris A. Saravanos

Ohio Aerospace Institute

Cleveland, Ohio

Dale A. Hopkins

Lewis Research Center

Cleveland, Ohio

Prepared for the 36th Structures, Structural Dynamics and Materials Conference cosponsored by AIAA, ASME, AHS, and ASC

New Orleans, Louisiana, April 10-13, 1995

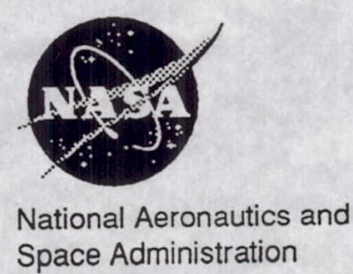

Space Administration 


\title{
MICROMECHANICS OF COMPOSITES WITH SHAPE MEMORY ALLOY FIBERS IN UNIFORM THERMAL FIELDS
}

\author{
Victor Birman \\ University of Missouri-Rolla \\ Engineering Education Center \\ 8001 Natural Bridge Road \\ St. Louis, MO 63121 \\ Dimitris A. Saravanos \\ Ohio Aerospace Institute \\ 22800 Cedar Point Road \\ Cleveland, Ohio 44142 \\ and \\ Dale A. Hopkins \\ National Aeronautics and Space Administration \\ Lewis Research Center \\ Cleveland, Ohio 44135
}

\begin{abstract}
Analytical procedures are developed for a composite system consisting of shape memory alloy fibers within an elastic matrix subject to uniform temperature fluxuations. Micromechanics for the calculation of the equivalent properties of the composite are presented by extending the multi-cell model to incorporate shape memory alloy fibers. A three phase concentric cylinder model is developed for the analysis of local stresses which includes the fiber, the matrix, and the surrounding homogenized composite. The solution addresses the complexities induced by the nonlinear dependence of the in-situ martensite fraction of the fibers to the local stresses and temperature, and the local stresses developed from interactions between the fibers and matrix during the martensitic and reverse phase transformations. Results are presented for a Nitinol/Epoxy composite. The applications illustrate the response of the composite in isothermal longitudinal loading and unloading, and in temperature induced actuation. The local stresses developed in the composite under various stages of the martensitic and reverse phase transformation are also shown.
\end{abstract}

\section{INTRODUCTION}

Shape memory alloys (SMAs) have found increasing applications as candidate smart materials and components of adaptive structures ${ }^{1}$. One potential application of shape memory alloys is their use as fibrous sensors or actuators within a composite material system. The development of such fibrous shape memory (SM) composites will provide weight savings, directionality in actuation and sensing, and some control over the resultant SM effect. Some technical details of such applications were discussed by Schetky and $\mathrm{Wu}^{2}$, Schetky ${ }^{3}$, and White et $\mathrm{al}^{4}$. The use of shape memory alloys as sensors may be related to the dependence of their properties on the martensite fraction (for example, their electric resistance) 5 . Conversely, the use of shape memory alloy (SMA) fibers as distributed micro-actuators is also very attractive because of the relatively high strains that can be generated.

In summary, the shape memory (SM) effect is associated with a martensitic phase transformation of the material from a parent phase (ref. 1). During such martensitic transformations, the change in crystallography results in substantial strains, called transformation strains. Of particular interest, are alloys which can recover their 
parent phase through a reverse phase transformation accompanied by recovery strains. These are SMAs which can undergo thermoelastic or complete phase transformations. In addition to the transformation strains, the phase transformations are accompanied by changes in the elastic, thermal and electric properties. The initiation of the martensitic or the reverse transformation depends on the free chemical energy in the material, which in turn, depends on the temperature and stress state. Hence, in a SM composite, the actuation process may be achieved by forcing the phase transformation to occur in the fibers by changing either the temperature or the stresses ${ }^{3}$ in the fibers.

Constitutive relations required to characterize the thermomechanical response of shape memory alloys were outlined in the works of Baumgart et $\mathrm{al}^{6}$, Tanaka ${ }^{7}$ and Sato, Tanaka ${ }^{8}$ and Brinson ${ }^{9}$. Applications of the unidirectional shape memory effect can be found in the papers of Liang and Rogers ${ }^{10}$, Lin and Rogers ${ }^{11}, \mathrm{Baz}$ et al ${ }^{12}$ and $\mathrm{Baz}$ and Chen ${ }^{13}$. Liang and Rogers ${ }^{14}$, Brinson and Lammering ${ }^{15}$ and Boyd and Lagoudas ${ }^{16}$ have provided formulations for the three-dimensional shape memory effect.

The analysis of composites incorporating continuous shape memory alloy fibers seems a rather open-ended research area involving solution of several problems including admissible micromechanics and the nonlinear constitutive relations of SMAs. Indeed, the properties and the induced strain of the SMA fibers depend on the fraction of martensite, which in turn, depends on the in-situ temperature and effective stress state. Because of the fiber-matrix interaction in the composite, the developed in-situ stresses will depend on phase transformation strains, thermal strains and the constituent properties. This interdependence results in a nonlinear thermomechanical response (even by assuming thermoelastic austenite and martensite phases), which makes an iterative analysis seemingly necessary, although as shown in the present paper, an analytical solution can be obtained in some cases without iterations. Numerous micromechanical theories may be used to formulate the present problem, yet, limited work has been reported in the area of SM composites. Three-dimensional micromechanics were reported by Boyd and Lagoudas ${ }^{16}$ using the Mori-Tanaka ${ }^{17}$ micromechanics model. This model yields equivalent properties of composites that are in close agreement with results obtained from the finite element analysis, as was shown by Noor and Shah ${ }^{18}$. However, the assumption of average constituent stresses/strains may be an area of limitation, considering the dependence of martensite fraction on local stress and temperature fields.

The present paper presents a combined micromechanical approach. A three-phase (SMA fiber, matrix, equivalent SM composite) concentric cylinder model is formulated for the accurate calculation of microstresses. The multi-cell micromechanics approach of Chamis and coworkers ${ }^{19}$ is also extended to predict the properties of the composite medium encompassing the SMA fibers and the matrix. The solution is obtained for prescribed values of the effective stress in the fiber which yield the martensite volume fraction and the properties of the fiber and the composite medium, thus, avoiding an iterative procedure associated with readjustment of the properties at each step. Results quantify the isothermal loading-unloading response of the composite under various temperature and initial stress conditions, the capability of the composite to undergo temperature induced actuation, and the resultant local stresses in the composite during a loading-unloading cycle with stress induced phase transformation in the fibers.

\section{CONSTITUTIVE EQUATIONS}

The assumed constitutive equations of the SMA fibers, the matrix and the resultant SM composite are presented in this section. The fibers and the matrix are assumed to remain within the linear elastic range, which is a reasonable assumption since plasticity seems to usually occur after the martensitic transformation. Both the fibers and matrix are assumed isotropic. However, the composite is orthotropic with a plane of symmetry transversely to the fibers.

Fibers.-Assuming that both martensitic and parent phases are isotropic and that there is no directionality in the alignment of the martensite and austenite crystals, the SMA fibers may be assumed to remain isotropic, and their engineering properties are linear functions of the martensite fraction (rule of mixtures) ${ }^{15-16}$,

$$
\left\{P_{f}\right\}=\xi\left\{P_{f}^{M}\right\}+(1-\xi)\left\{P_{f}^{A}\right\}
$$


where $P$ represents the thermoelastic properties, subscript $f$ indicates the fiber, $\xi$ is the current martensite volume fraction in the fiber, and superscripts $M$ and $A$ identify the martensite and austenite (parent) phases respectively.

The behavior of a shape memory alloy fiber during the martensite or reverse phase transformation can be represented in a differential form ${ }^{8}$,

$$
\dot{\mathrm{o}}_{f}=\left[C_{f}\right]\left\{\dot{\epsilon}-\alpha_{f} \dot{T}-\omega \dot{\xi}\right\}
$$

where: overdot indicates time derivatives, $s$ is the stress vector, $\mathrm{e}$ is the Green strain vector, $\mathrm{T}$ is the temperature, and $\mathrm{x}$ the martensite phase fraction; $[\mathrm{C}]$ and $\mathrm{a}$ are the current elasticity tensor and the vector of thermal expansion coefficients, respectively; $\omega$ is the phase transformation tensor relating the transformation strains to the martensite volume fraction. The phase transformation tensor represents the recovery strain limits ${ }^{10}$. In the case where the tensors in eq. (2) are linear functions of $\mathrm{x}$, eq. (2) can be integrated over time ${ }^{10.15}$ to yield:

$$
\begin{gathered}
\sigma_{f}-\sigma_{f}^{0}=\left[C_{f}(\xi)\right]\left\{\epsilon-\alpha_{f}(\xi) \Delta T-\omega \xi\right\}- \\
-\left[C_{f}(\xi)\right)\left\{\epsilon^{0}-\alpha_{f}(\xi)_{\Delta} T^{0}-\omega \xi^{0}\right\}
\end{gathered}
$$

In thermoelastic martensitic transformations, the martensite fraction depends on the free chemical energy in the material, which in turn, includes the thermal and strain energy components. This relation may be represented by the following phenomenological equations ${ }^{7,8}$ during the martensite transformation,

$$
\xi=1-\exp \left[b_{M}\left(T_{s}^{M}-T\right)+c_{M} \sigma_{e f f}\right]
$$

and during the reverse transformation,

$$
\xi=\exp \left[b_{A}\left(T_{s}^{A}-T\right)+c_{A} \sigma_{e f f}\right]
$$

$\mathrm{T}_{s}^{\mathrm{M}}$ and $\mathrm{T}_{s}^{\mathrm{A}}$ are the martensite and austenite phase start temperatures, $\sigma_{\text {eff }}$ is the effective stress, the coefficients $b_{M}$, $\mathrm{c}_{\mathrm{M}}, \mathrm{b}_{\mathrm{A}}$ and $\mathrm{c}_{\mathrm{A}}$ are given by,

$$
\begin{array}{ll}
b_{M}=\frac{\ln 0.01}{T_{s}^{M}-T_{f}^{M}} & c_{M}=\frac{b_{M}}{d_{M}} \\
b_{A}=\frac{\ln 0.01}{T_{s}^{A}-T_{f}^{A}} & c_{A}=\frac{b_{A}}{d_{A}}
\end{array}
$$

$\mathrm{T}_{\mathrm{F}}^{\mathrm{M}}$ and $\mathrm{T}_{\mathrm{F}}^{\mathrm{A}}$ are the martensite and austenite finish temperatures, and $\mathrm{d}_{\mathrm{M}}$ and $\mathrm{d}_{\mathrm{A}}$ are constants defining the slopes of the martensitic and reverse transformation lines, respectively. The characteristic temperatures depend also on the effective stress: 


$$
\begin{aligned}
& <\bar{T}_{s}^{M}, \bar{T}_{f}^{M}>=<T_{s}^{M}, T_{f}^{M}>+\frac{\sigma_{e f f}}{d_{M}} \\
& \left.<\bar{T}_{s}^{A}, \bar{T}_{f}^{A}\right\rangle=<T_{s}^{A}, T_{f}^{A}>+\frac{\sigma_{e f f}}{d_{A}}
\end{aligned}
$$

where overbar indicates the start and finish temperatures of the respective phase transformation under reference stress-free conditions. Eqs. (4) and (5) may be rearranged to provide the range of the effective stress where stress induced martensitic and reverse phase transformations (at a prescribed temperature) will occur, respectively:

$$
\begin{aligned}
& \frac{b_{M}}{c_{M}}\left(T-T_{s}^{M}\right) \leq \sigma_{e f f} \leq \frac{\ln 0.01}{c_{M}}+\frac{b_{M}}{c_{M}}\left(T-T_{s}^{M}\right) \\
& \frac{\ln 0.01}{c_{A}}+\frac{b_{A}}{c_{A}}\left(T-T_{s}^{A}\right) \leq \sigma_{e f f} \leq \frac{b_{A}}{c_{A}}\left(T-T_{s}^{A}\right)
\end{aligned}
$$

Note that in accordance with the metallurgical approach, eqs. (8) and (9) are obtained by identifying the values $\xi=1$. and $\xi=0.01$ with the completion of the martensite and reverse transformations, respectively.

Matrix.-The matrix is assumed to be isotropic and elastic, such that:

$$
\sigma_{m}=C_{m}\left(\epsilon-\alpha_{m} \Delta T\right)
$$

where $\Delta \mathrm{T}$ is the difference between the current and stress-free temperatures, and subscript $\mathrm{m}$ indicates the matrix.

Composite.-The equivalent constitutive equations for a composite medium with SMA fibers undergoing a martensitic or reverse transformation can be postulated to have the following form

$$
\dot{\sigma}_{c}=\left[C_{c}\right]\left\{\dot{\epsilon}-\alpha_{c} \dot{T}-\omega_{c} \dot{\xi}\right\}
$$

where subscript $c$ indicates the homogenized composite and $x$ is the martensite phase fraction in the fibers. The main difference with eq. (2) is that the resultant composite will exhibit orthotropy in the LT plane (see Fig. 1) including the components of the phase transformation vector:

$$
\omega_{c}=\left\{\omega_{c L}, \omega_{c T}, \omega_{c T}\right\}^{T}
$$

Within a range of linear dependence of the elastic, thermal and transformation components to the martensite volume fraction, integration of eq. (11) over time may yields,

$$
\begin{gathered}
\sigma_{c}-\sigma_{c}^{0}=C_{c}(\xi)\left[\epsilon-\alpha_{c}(\xi) \Delta T-\omega_{c} \xi\right]- \\
\left.-C_{c}(\xi)\right]\left[\epsilon^{0}-\alpha_{c}\left(\xi^{0}\right) \Delta T^{0}-\omega_{c} \xi^{0}\right]
\end{gathered}
$$


Both eqs. (3) and (13) remain valid during the martensitic and reverse transformation and they reflect the behavior of thermoelastic shape memory alloy materials stressed below their second yield limit associated with the plastic slip of the martensitic phase $e^{20}$. It is pointed out, that eq. (13) represents rather an approximation, because the transverse properties of the composite are not linear functions of $\mathrm{x}$. In this case linearization over a range of $\mathrm{x}$ may be required, and eq. (13) should be applied within this range. In all cases, the initial conditions corresponding to the particular phase of the transformation should be considered, moreover, the continuity of stress-strain curves at the boundaries of adjacent transformation phases is preserved. It is finally emphasized that although equations (3) and (13) can describe both martensitic and reverse transformations, the corresponding stress-strain curves do not overlap each other, because of the differences between the effective start and finish stresses and temperatures defining the onset and completion of the martensite and reverse phase transformations [eqs. (4-5) and (8-9)].

\section{MICROMECHANICS}

\section{Multi-Cell Approach}

Good approximations of the equivalent properties of the previous SMA composite may be obtained using the multi-cell micromechanics approach ${ }^{19}$. According to the method, the representative cell is divided to a set of subregions (see Fig. 1a), each one containing portions of a square SMA fiber and the matrix. It is further assumed that the strains/stresses in the various regions of the representative cell are constant, the Poisson's effect on transverse deformations is negligible, and the fibers are packed in a square array formation. The analysis employs a mechanics of materials approach based on the equilibrium of stresses within each region and the compatibility of strains at the boundaries of each region. Thus, the equivalent incremental constitutive relations of the SMA composite are derived in accordance eq. (11).

The resultant expressions for the elastic and thermal properties are similar to the ones reported for thermoelastic composites, hence, are shown in the Appendix. According to the differential form of the constitutive relations for the fibers (2) and the composite (11), the phase transformation/recovery strain component has an analogous form to the thermal strain component. Therefore, at a given temperature and martensite fraction in the fibers, the equivalent transformation tensor of the composite $\mathrm{w}_{\mathrm{c}}$ may be evaluated similarly to the thermal expansion coefficient of the composite (see Appendix).

$$
\begin{aligned}
& \omega_{c L}=k_{f} \omega_{f} \frac{E_{f}}{E_{L}} \\
& \omega_{c T}=\frac{k_{f} \omega_{f}}{1-\sqrt{k_{f}}\left(1-\frac{E_{m}}{E_{f}}\right)} \frac{E_{m}}{E_{T}}
\end{aligned}
$$

where, subscripts $\mathrm{L}$ and $\mathrm{T}$ indicate the longitudinal and transverse direction in the composite with respect to the fibers, $k_{f}$ is the fiber volume fraction, $E_{q}$ and $E_{m}$ are the moduli of elasticity of the fiber and matrix respectively, and $\mathrm{E}_{\mathrm{L}}$ and $\mathrm{E}_{\mathrm{T}}$ are the longitudinal and transverse moduli of elasticity of the composite medium.

\section{Concentric Cylinder Approach}

The previous multi-cell micromechanics is expected to yield sufficient approximations of the equivalent properties of the composite. Considering, however, the potential significance of local stresses (microstresses) in the response of the SMA fibers, a concentric cylinder model can provide better representations of the local stresses, which may have an effect on the axial response of the SMA composite. The three-cylinder assembly model shown in Fig. $1 \mathrm{~b}$ was formulated containing a fiber, surrounded by a matrix and an infinite SM composite medium with constitutive relations provided by either eqs. (11) or (13) and equivalent properties provided by the previously 
described multi-cell micromechanics. The inclusion of the infinite composite cylinder is important, because it introduces the interactions between the fiber and matrix, and the surrounding composite due to the mismatch in the respective elastic, thermal and phase transformation components. Moreover, the consideration of a surrounding infinite composite, instead of a surrounding infinite matrix, extends the application of the approach beyond the low fiber volume ratio regime.

Considering the axisymmetry of the problem, the equations of equilibrium within each material region of the concentric cylinder model (fiber, matrix, and composite) are reduced to the following equation:

$$
\frac{d \sigma_{r}}{d r}+\frac{\sigma_{r}-\sigma_{\theta}}{r}=0
$$

where subscripts $x, r$ and $\theta$ indicate longitudinal, radial and tangential directions, respectively. The axial strain is assumed to remain constant throughout the cell and the composite, while the tangential and radial strains are given by

$$
\epsilon_{\theta}=\frac{w}{r} \quad \epsilon_{r}=\frac{d w}{d r}
$$

where $w$ is the radial displacement. Eqs. (16) imply that the range of validity of the present solution is confined to geometrically linear deformations.

Combinations of eqs. (15) and (16) and then substitution of constitutive eqs. (3), (10) and (13) for the fiber, matrix and composite regions respectively, yield an admissible solution in the form:

$$
\left\{\begin{array}{c}
w_{f} \\
w_{m} \\
w_{c}
\end{array}\right\}=\left\{\begin{array}{c}
A_{1}^{f} \\
A_{1}^{m} \\
A_{1}^{c}
\end{array}\right\} r+\left\{\begin{array}{c}
A_{2}^{f} \\
A_{2}^{m} \\
A_{2}^{c}
\end{array}\right\} r^{-1}
$$

where $A_{k}{ }^{j}$ are constants of integration. The requirements of a bounded solution yield $A_{1}{ }^{c}=A_{2}{ }^{f}=0$. The remaining constants of integration are related via the conditions of continuity in the displacements and stresses at the fibermatrix and matrix-composite interfaces:

$$
\begin{aligned}
w^{f}\left(r_{f}\right) & =w^{m}\left(r_{f}\right) \\
\sigma_{r}^{f}\left(r_{f}\right) & =\sigma_{r}^{m}\left(r_{f}\right) \\
w^{m}\left(r_{m}\right) & =w^{c}\left(r_{m}\right) \\
\sigma_{r}^{m}\left(r_{m}\right) & =\sigma_{r}^{c}\left(r_{m}\right)
\end{aligned}
$$

where $r_{f}$ and $r_{m}$ are the radius of the fiber and the external matrix interface (Fig. 1b), respectively.

The effective stress, required for the determination of the martensitic fraction [eqs. (8-9)] and the state of the composite, is the following function of the fiber stresses:

$$
\sigma_{e f f}=\left\{0.5\left[\left(\sigma_{x}^{f}-\sigma_{\theta}^{f}\right)^{2}+\left(\sigma_{x}^{f}-\sigma_{r}^{f}\right)^{2}+\left(\sigma_{r}^{f}-\sigma_{\theta}^{f}\right)^{2}\right\}^{1 / 2}\right.
$$

It is pointed out that the solution yields equal tangential and radial strains in the in the fiber, that is, $\epsilon_{\theta}=\epsilon_{\mathrm{r}}=\mathrm{A}_{1}{ }_{\mathrm{f}}$. Considering the isotropy in the transverse plane of the fiber, this also implies that the radial and tangential stresses in the fiber are equal and remain constant along the radial direction. This simplifies further the expression for the effective stress in the fibers: 


$$
\left(\sigma_{e f f}\right)_{f}=\left|\sigma_{x}^{f}-\sigma_{r}^{f}\right|_{f}
$$

\section{Computational Procedure}

As seen in the previous analytical formulation, the analysis of both the properties and the local stresses in a fibrous SM composite may require an incremental and iterative approach. This is due to the fact that the properties of the fibers, and, therefore, the average properties of the composite depend on the stresses via the martensite fraction. In turn, the stresses are affected by the properties of the constituent materials. To further complicate the matter, the start and finish temperatures of the martensitic and reverse transformation depend also on the stresses.

However, the iterative process can be avoided and solution may be obtained in one step, within the range of $x$ where eq. (13) is valid. In the later approach, the analysis begins with predefined values of the temperature and the effective stress in the fiber. Then the martensite fraction in the fibers is calculated according to eqs. (4) or (5). Once the martensitic function is defined, the properties of the fibers and the equivalent properties of the surrounding composite are calculated. Then, eqs. (15) and (20) are solved and the constants of integration $A_{1}{ }^{f}, A_{1}{ }^{m}, A_{2}{ }^{m}, A_{2}{ }^{c}$ and the axial strain $\epsilon_{x}$ corresponding to the effective stress are found. The local strains and stresses are calculated. $A$ final integration over the radius yields the average longitudinal stress in the composite

$$
\sigma_{c x}=\int_{0}^{r_{m}} \sigma_{x} d r
$$

\section{APPLICATION CASES}

The application cases presented in the following paragraphs illustrate the predicted stress-strain response of the composite under isothermal loading and unloading conditions, the capability of the composite to induce axial strains as a result of variations in the temperature, and the developed local stress fields in the material during stress induced martensitic and reverse transformations in the fiber. The shape memory material considered in the examples is nitinol $\mathrm{Ni}-44.8 \mathrm{wt} \% \mathrm{Ti}$ whose properties are presented in Table 1 as reported by Lei and $\mathrm{Wu}^{21}$. An epoxy matrix was assumed with properties shown in Table 1 and the fiber volume fraction was taken equal to 0.3.

\section{Composite Response}

The predicted loading and unloading cycle of the SM composite at $40^{\circ} \mathrm{C}$ is shown in Fig. 2. It is assumed that all local non-mechanical (shape memory and thermal) stresses are zero when the fibers are in the austenite phase (point A), that is, matrix consolidation occurs at $40^{\circ} \mathrm{C}$ with austenite fibers and the thermal residual stresses are zero. As seen in Fig. 2, the composite deforms linearly from point A to B because the fibers remain in the austenite phase $(x=0)$. After point $C$ the effective stress in the fiber becomes sufficiently high for the martensite transformation to start and to induce transformation strains in the fibers, thus resulting in the nonlinear segment $B C(0<x<1)$. At point $C$ the fibers are fully transformed to the martensite phase $(x=1)$ and the subsequent linear section $C D$ illustrates the behavior of the composite with elastic martensite fibers. The different slopes of sections $A B$ and $C D$ represent the differences in the moduli of the martensite and austenite phases of the fiber. The predicted unloading phase is shown in section DEF. As the stress is reduced, the fibers remain in the martensitic phase until the reverse transformation starts (point $E$ ). Further stress reductions after point $E$, reduce the martensite fraction in the fibers and the recovery strains, resulting in the nonlinear curve $E F(1>x>0)$. After point $F$ the fibers are fully transformed to their parent phase $(x=0)$ and the subsequent unloading follows the linear path FA. 
The isothermal unloading of the previous SM composite system (reverse transformation in the SMA fibers) at four different temperatures is shown in Fig. 3. As in the previous case, the stress-free condition in the composite occurs with austenite fibers at $40^{\circ} \mathrm{C}$. As seen in Fig. 3, at higher temperatures higher longitudinal stresses are required for starting and finishing the reverse transformation in the fibers. Fig. 3 implicitly illustrates the potential of SM composites to be used as strain or stress actuators by varying the temperature of the composite, as well as, it quantifies the ranges of stress, strain and temperature where such actuation is feasible. Within this range, the composite has the potential to generate significant longitudinal deformations or stresses. In all cases shown in Fig. 3, high recovery strains of an order of 6-8\% have been calculated. Outside this range, the SM composite can not act as an actuator. However, as shown in the following case, it seems that this range may be modified by changing the processing conditions.

Figure 4 also shows the isothermal unloading of the composite, however, matrix consolidation with fibers in the martensitic phase was assumed, such that the stress-free condition corresponds to fully transformed martensite fibers at $40^{\circ} \mathrm{C}$. In this manner, different states of local stresses are developed in the composite compared with the previous cases, that is, tensile longitudinal stresses in the fibers as the reverse transformation progresses. The predicted composite behavior differs significantly from the curves in Fig. 3. The reverse transformation occurs at different stress and strain ranges, and the recovery strains are smaller, of an order of $4 \%$ or less. This illustrates that the range of actuation of these novel composites may be modified by controlling the initial stresses in the composite. The predicted nonlinear unloading curve is also non-monotonic with respect to the stress. This is possible, because on the onset of reverse transformation tensile longitudinal and radial stresses will develop in the fiber (due to the negative transformation strain), which may suffice or exceed the values required to maintain a self-sustained reverse transformation. Hence, in order to achieve monotonic decrease in the martensite fraction (and the corresponding effective stress) in the fibers, the axial stress needs to increase and decrease again. This type of behavior is expected be observed in a "strain controlled" uniaxial test.

The predicted capability of the composite to induce axial strain, under zero axial stress conditions, by controlling the temperature in the composite is shown in Fig. 5. The curve corresponds to a composite with stressfree austenite fibers. The curve begins at $20^{\circ} \mathrm{C}$ when the fibers are in the martensitic phase. The reverse transformation starts at $30^{\circ} \mathrm{C}$ and finishes at $55^{\circ} \mathrm{C}$ when the fibers have been fully transformed into austenite. It was predicted that, a $25^{\circ} \mathrm{C}$ variation in temperature will result in a recovery strain of $6.3 \%$.

\section{Local Stresses}

The predicted local stress distributions in the composite are presented here. The calculation of the stresses in the fiber and the matrix is important to ensure the integrity of the composite and prevent microcracking and fiber-matrix debonding. The longitudinal, radial and hoop stresses are shown in Figs. 6-9 as functions of the normalized radius for various phases of the isothermal stress-strain cycle shown in Fig. 2. The normalized radius in the figures is defined as the ratio of the local radius to the radius of the fiber $\left(\mathrm{r} / \mathrm{r}_{\mathrm{f}}\right)$.

Figure 6 shows the stresses in the composite, when an average longitudinal stress $s_{c x}=-53.6 \mathrm{MPa}$ is applied and the fibers still remain in the austenitic phase. Fig. 7 shows the stresses in the composite when the fibers undergo martensitic transformation $(\xi=0.83)$ under the application of a tensile stress $\left(s_{c x}=176 \mathrm{MPa}\right)$. The applied tensile stress results in tensile longitudinal stresses in both the fiber and the matrix and in a tensile hoop stress in the matrix. Fig. 8 shows the local stress in the composite with fully transformed fibers $\left(x=1, s_{c x}=288 \mathrm{MPa}\right.$ ). In spite the significant increase in the average applied stress, there is little change in the magnitudes of the radial and hoop stresses, but the longitudinal stresses in the fibers and the matrix increase significantly.

Predicted stress distributions corresponding to the reverse phase transformation in the fibers $\left(x=0.40, s_{c x}=\right.$ $12 \mathrm{MPa}$ ) are shown in Fig. 9. A comparison indicates that the stresses tend to return to a distribution similar to the one shown in Fig. 6. Further reduction of the longitudinal stress to $-53.6 \mathrm{MPa}$ resulted in full recovery of the parent phase in the fibers $(x=0)$ and yielded a stress distribution identical to the one shown in Fig. 6 . This is not surprising, because at this point both loading and unloading curves coincide, hence, the corresponding internal stress states in the composite should be identical. 


\section{SUMMARY}

Micromechanics for the evaluation of the properties and analysis stress in unidirectional composite systems with continuous shape memory alloy fibers in an elastic matrix were presented. The multi-cell micromechanical method was extended to calculate the average properties of the composite when the fibers undergo martensite or reverse phase transformations. The axial response of the composite and the local stresses in the fiber and the matrix were determined using a concentric cylinder model, where the fiber and the surrounding matrix are embedded within an infinite composite medium.

Evaluations of the mechanics were presented for a Nitinol/Epoxy composite system. The numerical results demonstrated the longitudinal response of the composite under isothermal loading and unloading conditions. They also illustrated the capability of the composite to induce substantial longitudinal strains under variation of temperature. The ranges of stress, strain and temperature where martensite and reverse phase transformations occur were also shown. It was predicted that substantial actuation strains may be induced in composites of intermediate fiber volume fraction $(-0.3)$. It was also shown, that the range of stress and strain where the composite may be used as actuator may be modified by controlling the stress-free conditions in the composite. Finally, the distribution of microstresses was considered for an entire isothermal loading-unloading cycle of the composite material with stress induced phase transformation. The characteristic distribution of stresses remained unaffected, but their magnitude and sign changed drastically, depending on the martensite fraction of the fibers and the applied external load. In closing, this work has been the first step towards understanding and modelling the complex, yet promising, active response of continuous SMA fiber composites. Additional research will be conducted and reported in the future.

\section{ACKNOWLEDGEMENT}

This research was conducted during an ASEE summer faculty fellowship of Dr. Victor Birman at the NASA Lewis Research Center.

\section{REFERENCES}

1. Duerig, T.W., Melton, K.N., Stokel, D. and Wayman, C.M., Engineering Aspects of Shape Memory Alloys, Butterworth-Heinemann, London, 1990.

2. Schetky, L.McD. and Wu, M.H, "The Properties and Processing of Shape Memory Alloys for Use as Actuators in Intelligent Composite Materials", Smart Structures and Materials, Eds. Haritos, G.K. and Srinivasan, A.V., ASME, New York, 1991, pp. 65-71.

3. Schetky, L.McD., "The Role of Shape Memory Alloys in Smart/Adaptive Structures", Shape-Memory Materials and Phenomena -Fundamental Aspects and Applications, Eds. Liu, C.T. et. al, Materials Research Society Symposium Proceedings, Vol. 246, Materials Research Society, Pittsburgh, 1992, pp. 299-307.

4. White, S.R., Whitlock, M.E., Ditman, J.B. and Hebda, D.A., "Manufacturing of Adaptive Graphite/Epoxy Structures with Embedded Nitinol Fibers", Adaptive Structures and Material Systems, Eds. Carman, G.P. and Garcia, E., ASME, New York, 1993, pp. 71-79.

5. Airoldi, G., Riva, G., Ranucci, T. and Vicentini, B., "Electric Transport Properties of a NiTi Shape Memory Alloy Under Applied Stress", Shape-Memory Materials and Phenomena -Fundamental Aspects and Applications, Eds. Liu, C.T. et al, Materials Research Society Symposium Proceedings, Vol. 246, Materials Research Society, Pittsburgh, 1992, pp. 277-281.

6. Baumgart, F., Jorde, J. and Reiss, H.G., "Memory Alloys - Properties, Phenomenological Theory and Applica tions", NASA TM-77904, Translation from German: Technische Mitteilunger Krupp, Forschungsberichte, Vol. 34, July 1976.

7. Tanaka, K., "A Thermomechanical Sketch of Shape Memory Effect: One Dimensional Tensile Behavior", Res Mechanica, Vol. 18, 1986, pp. 251-263.

8. Sato, Y. and Tanaka, K., "Estimation of Energy Dissipation in Alloys due to Stress-induced Martensitic Transformation", Res Mechanica, Vol. 23, 1988, pp. 381-393. 
9. Brinson, L. C., "One-Dimensional Constitutive Behavior of Shape Memory Alloys: Thermomechanical Derivation with Non-Constant Material Functions and Redefined Martensite Internal Variable," Journal of Intelligent Material Systems and Structures, Vol. 4. April 1993, pp. 229-242.

10. Liang, C. and Rogers, C.A., "One-Dimensional Thermomechanical Constitutive Relations for Shape Memory Materials", Journal of Intelligent Systems and Structures, Vol. 1, 1990, pp. 207-234.

11. Lin, M.W. and Rogers, C.A., "Analysis of Stress Distribution in a Shape Memory Alloy Composite Beam", AIAA Paper 91-1164-CP, 1991.

12. Baz, A., Imam, K. and McCoy, J., "Active Vibration Control of Flexible Beams Using Shape Memory Actuators", Journal of Sound and Vibration, Vol. 140, 1990, pp. 437-456.

13. Baz, A. and Chen, T., "Performance of Nitinol-Reinforced Drive Shafts", Smart Structures and Intelligent Systems, Eds. Hagood, N.W. and Knowles, G.J., Vol. 1917, Part II, SPIE, 1993, pp. 791-808.

14.Liang, C. and Rogers, C.A., "The Multi-Dimensional Constitutive Relations of Shape Memory Alloys", AIAA Paper 91-1165-CP, 1991.

15. Brinson, L.C. and Lammering, R., "Finite Element Analysis of the Behavior of Shape Memory Alloys and Their Ap plications", International Journal of Solids and Structures, Vol. 30, 1993, pp. 3261-3280.

16. Boyd, J.G. and Lagoudas, D.C., "Thermomechanical Response of Shape Memory Composites", Smart Structures and Intelligent Systems, Eds. Hagood, N.W. and Knowles, G.J., Vol. 1917, Part II, SPIE, 1993, pp. 774-79 0.

17. Mori, T. and Tanaka, K., "Average Stress in Matrix and Average Elastic Energy of Materials with Misfitting Inclusions", Acta Metallurgica, Vol. 21, 1973, pp. 571-574.

18. Noor, A.K. and Shah, R.S., "Effective Thermoelastic and Thermal Properties of Unidirectional Fiber-Reinforced Composites and Their Sensitivity Coefficients", Composite Structures, Vol. 26, 1993, pp. 7-23.

19. Chamis, C.C., "Simplified Composite Micromechanics Equations for Hygral, Thermal and Mechanical Properties", NASA TM-83320, 1983.

20. Wayman, C.M. and Duerig, T.W., "An Introduction to Martensite and Shape Memory", in Engineering Aspects of Shape Memory Alloys, Eds. Duerig, T.W., et al, Butterworth-Heinemann, London, 1990, pp. 3-2019.

21. Lei, C.Y. and Wu, M.H., "Thermomechanical Properties of NiTi-base Shape Memory Alloys", Smart Structures and Materials, Eds. Haritos, G.K. and Srinivasan, A.V., ASME, New York, 1991, pp. 73-77. 


\section{APPENDIX}

Elastic Properties of SM composites using the multi-cell method ${ }^{19}$ :

$$
\begin{gathered}
E_{L}=E k_{f}+E_{m} k_{m} \\
E_{T}=E_{m}\left[1-\sqrt{k_{f}}+\frac{\sqrt{k_{f}}}{1-\sqrt{k_{f}\left(1-\frac{E_{m}}{E_{f}}\right)}}\right] \\
G_{L T}=G_{m}\left[\begin{array}{c}
\left.1-\sqrt{k_{f}}+\frac{\sqrt{k_{f}}}{1-\sqrt{k_{f}}\left(1-\frac{G_{m}}{G_{f}}\right)}\right] \\
G_{T T}=\frac{G_{m}}{1-\sqrt{k_{f}}\left(1-\frac{G_{m}}{G_{f}}\right)} \\
v_{L T}=v_{f} k_{f}+v_{m} k_{m} \\
v_{T T}=\frac{E_{T}}{2 G_{T T}}-1
\end{array}\right.
\end{gathered}
$$

Coefficients of Thermal Expansion:

$$
\begin{gathered}
\alpha_{L}=\left(E_{f} \alpha k_{f}+E_{m} \alpha_{m} k_{m}\right) / E_{L} \\
\alpha_{T}=\frac{E_{m}}{E_{T}}\left[\alpha_{m}\left(1-\sqrt{k_{f}}\right)+\sqrt{k_{f}} \frac{\alpha_{f} \bar{k}_{f}+\alpha_{m}\left(1-\sqrt{k_{f}}\right)}{1-\sqrt{k_{f}}\left(1-\frac{E_{m}}{E_{f}}\right)}\right]
\end{gathered}
$$

where $E_{m}, G_{m}, v_{m}$ and $\alpha_{m}$ are the normal and shear moduli of elasticity, the Poisson's ratio and the coefficient of thermal expansion of the matrix, respectively; and $n_{f}$ is the Poisson's ratio of the fiber which is independent of the martensite fraction. Volume fractions of the fiber and the matrix are denoted by $k_{f}$ and $k_{m}$, respectively. Subscripts $L$ and $\mathrm{T}$ identify the longitudinal and transverse directions. 
Table 1

Properties of Nitinol fibers ${ }^{21}$

(Annealing temperature $600^{\circ} \mathrm{C}$ )

\begin{tabular}{|c|c|c|c|}
\hline Property & & Nitinol & Epoxy \\
\hline $\begin{array}{l}\mathrm{E}^{\mathrm{A}} \\
\mathrm{E}^{\mathrm{M}} \\
\nu \\
\mathrm{a}^{\mathrm{A}} \\
\mathrm{a}^{\mathrm{M}} \\
\mathrm{T}^{\mathrm{M}}{ }^{\mathrm{S}} \\
\mathrm{T}^{\mathrm{M}}{ }^{\mathrm{f}} \\
\mathrm{T}^{\mathrm{A}}{ }^{\mathrm{s}} \\
\mathrm{T}^{\mathrm{A}^{\mathrm{s}}} \\
\mathrm{d}_{\mathrm{M}} \\
\mathrm{d}_{\mathrm{A}} \\
\omega\end{array}$ & $\begin{array}{l}\text { (GPa) } \\
(\mathrm{GPa}) \\
\left(10^{-6} \rho^{\circ} \mathrm{C}\right) \\
\left(10^{-6} \%^{\circ} \mathrm{C}\right) \\
\left({ }^{\circ} \mathrm{C}\right) \\
\left({ }^{\circ} \mathrm{C}\right) \\
\left({ }^{\circ} \mathrm{C}\right) \\
\left({ }^{\circ} \mathrm{C}\right) \\
\left(\mathrm{MPa} /{ }^{\circ} \mathrm{C}\right) \\
\left(\mathrm{MPa}{ }^{\circ} \mathrm{C}\right)\end{array}$ & $\begin{array}{l}30.0 \\
13.0 \\
0.33 \\
12.5 \\
18.5 \\
23 \\
5 \\
29 \\
51 \\
11.3 \\
4.5 \\
0.07\end{array}$ & $\begin{array}{c}3.4475 \\
- \\
0.35 \\
64.8 \\
- \\
- \\
- \\
- \\
- \\
- \\
- \\
-\end{array}$ \\
\hline
\end{tabular}




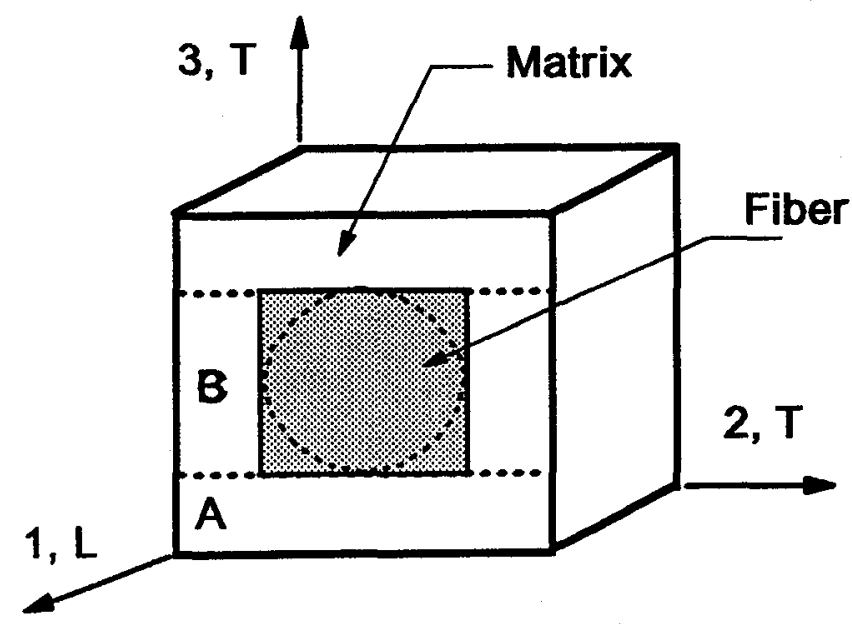

A: Matrix Region

B: Matrix and Fiber Region

(a) Multi-Cell Model

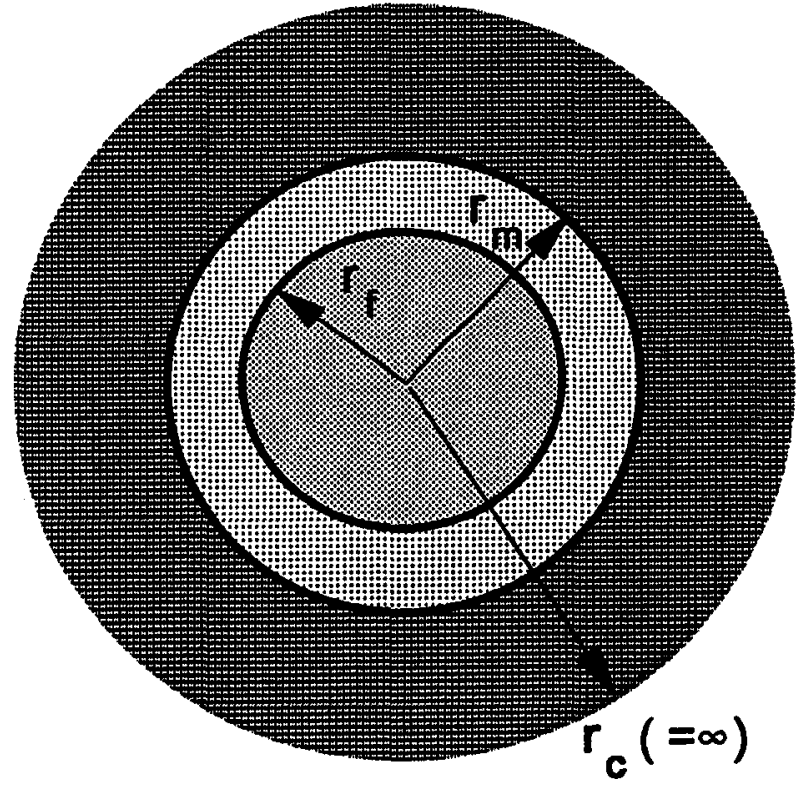

(b) Concentric Cylinder Model

Fig. 1 Representative composite models. (a) Multi-cell; (b) Concentric cylinders. 


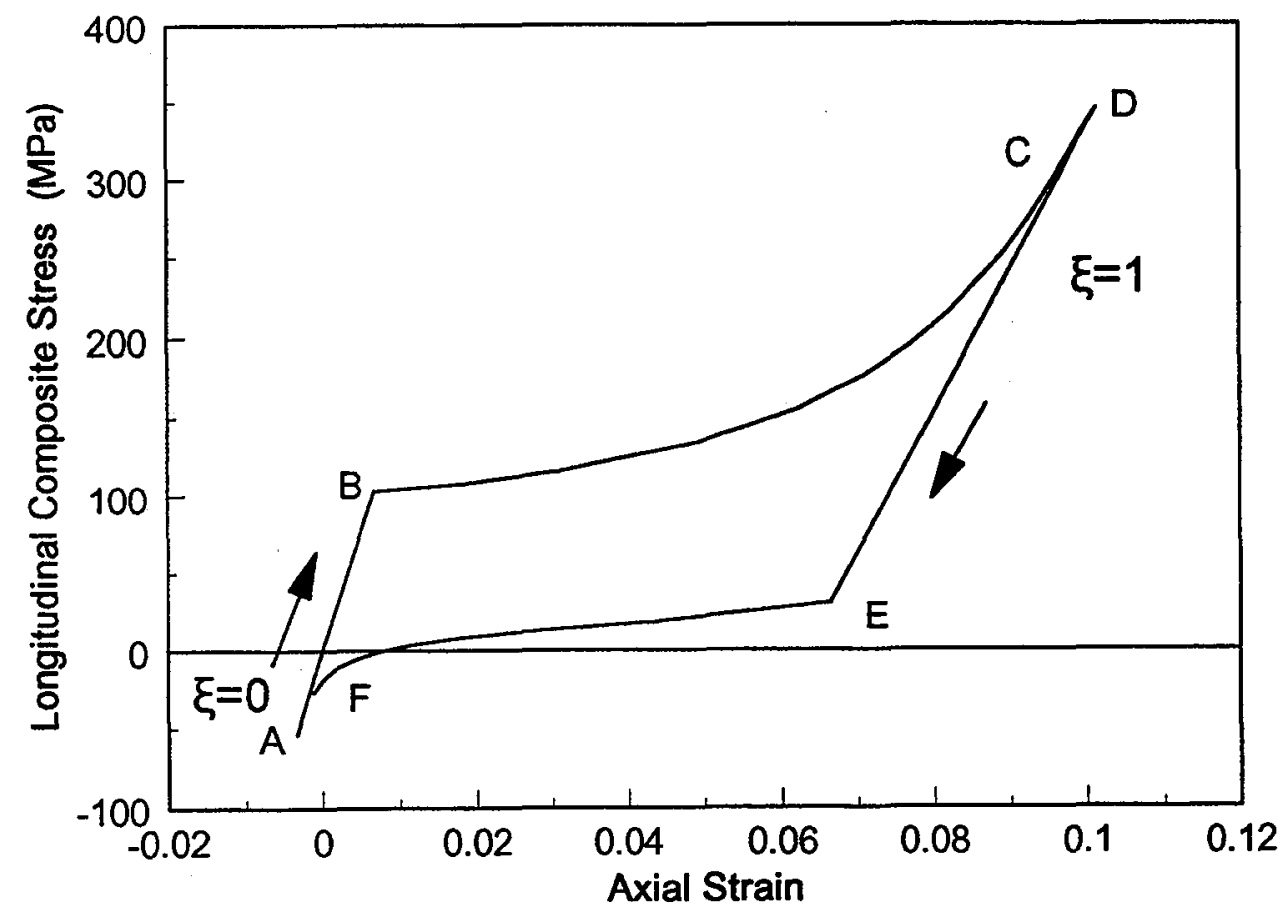

Fig. 2 Isothermal stress-strain cycle of $30 \%$ FVR nitinol/epoxy composite (40 degs. C). Stress free conditions correspond to austenite fibers.

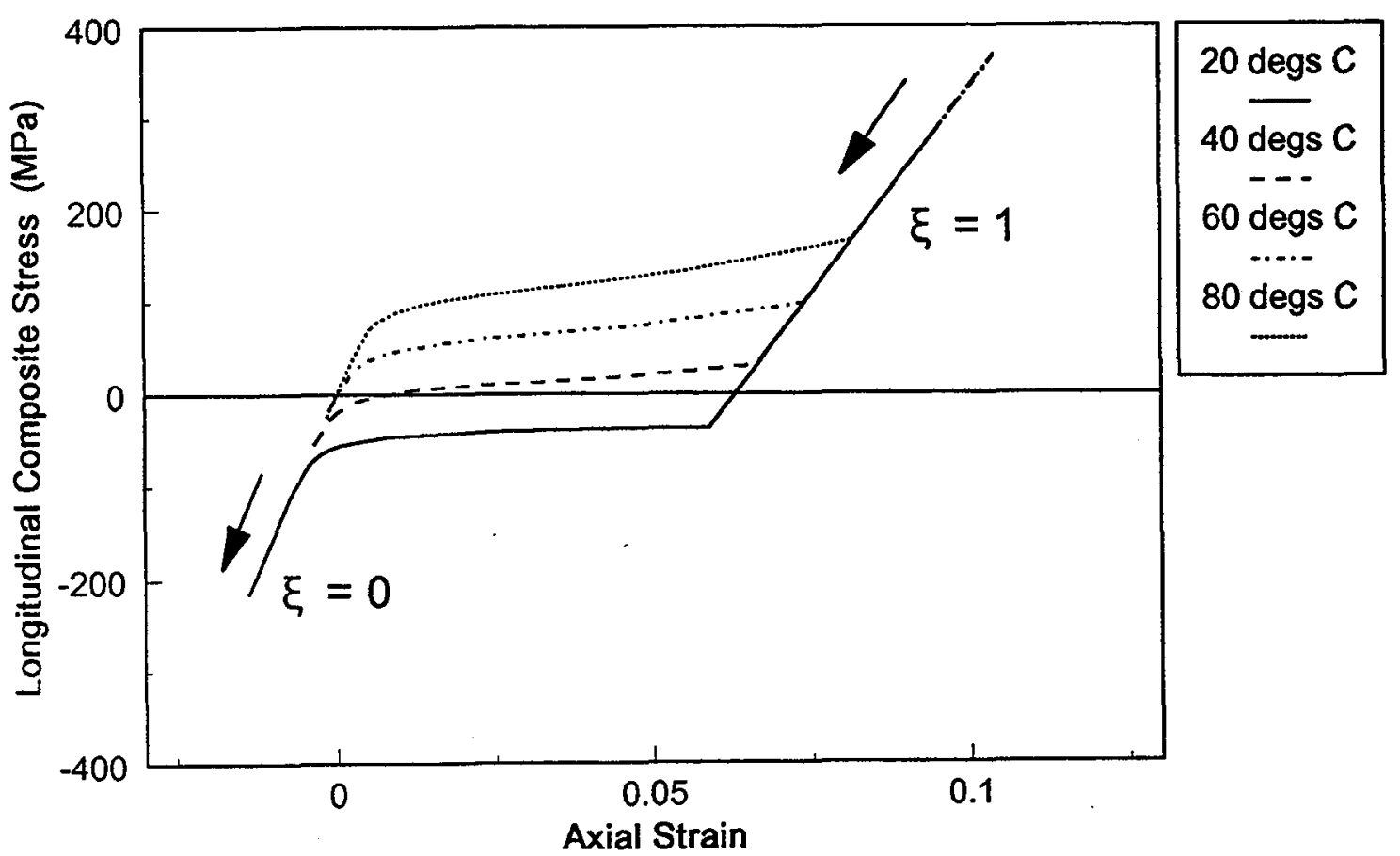

Fig. 3 Isothermal unloading of 30\% FVR nitinol/epoxy composite at various temperatures. Stress free conditions correspond to austenite fibers. 


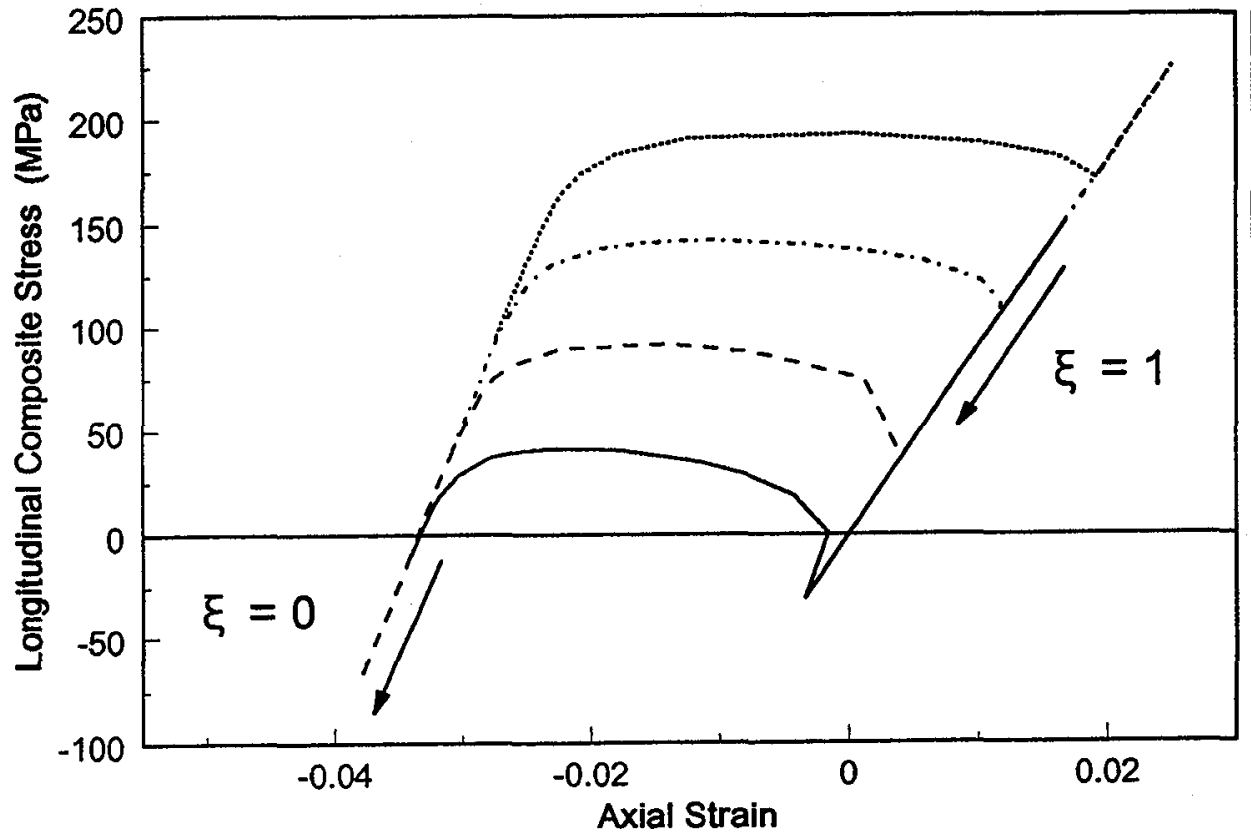

20 degs C

40 degs $C$

60 degs $C$

.....

80 degs $C$

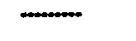

Fig. 4 Isothermal unloading of 30\% FVR nitinol/epoxy composite at various temperatures. Stress-free conditions correspond to martensite fibers.

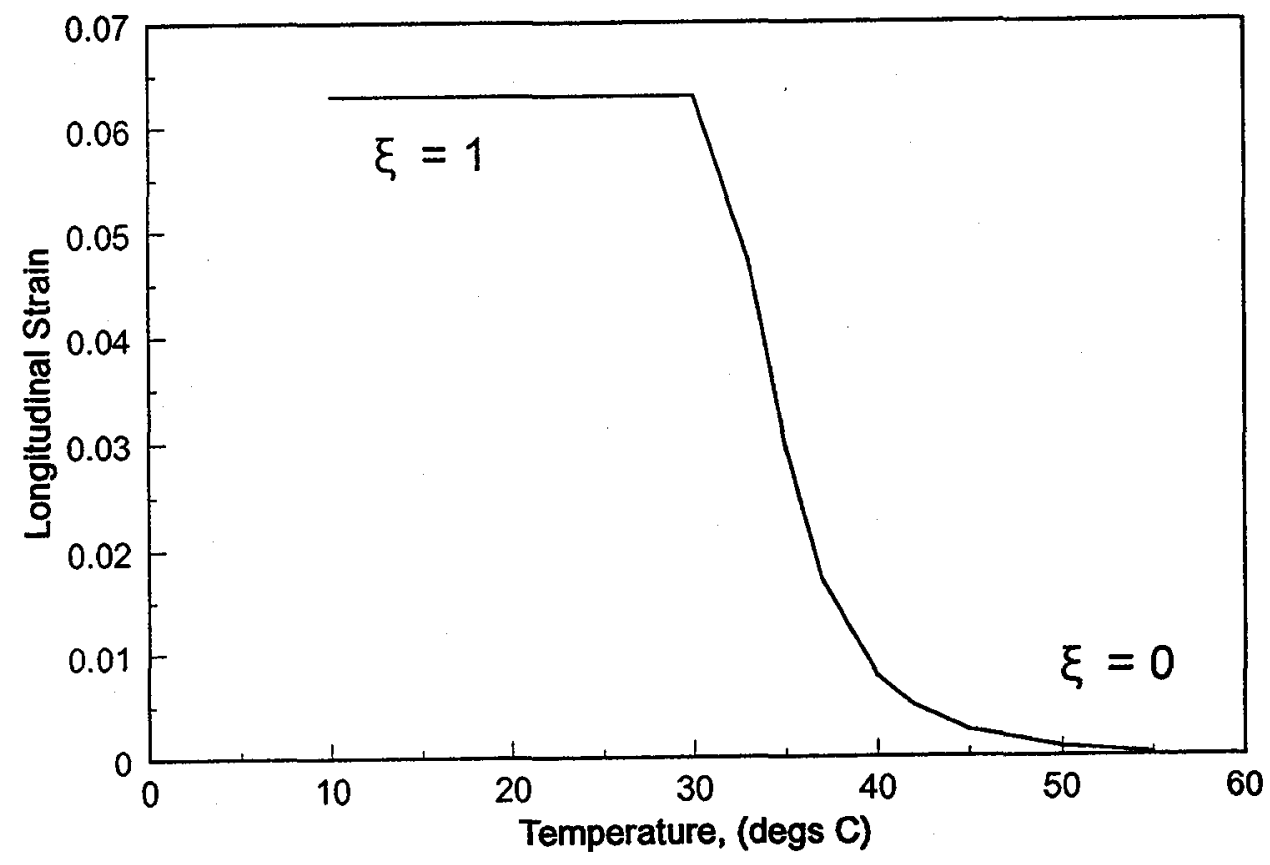

Fig. 5 Temperature induced axial strain actuation with of $30 \%$ FVR nitinol/epoxy composite with reverse transformation in the fibers. Composite remains under stress-free conditions. 

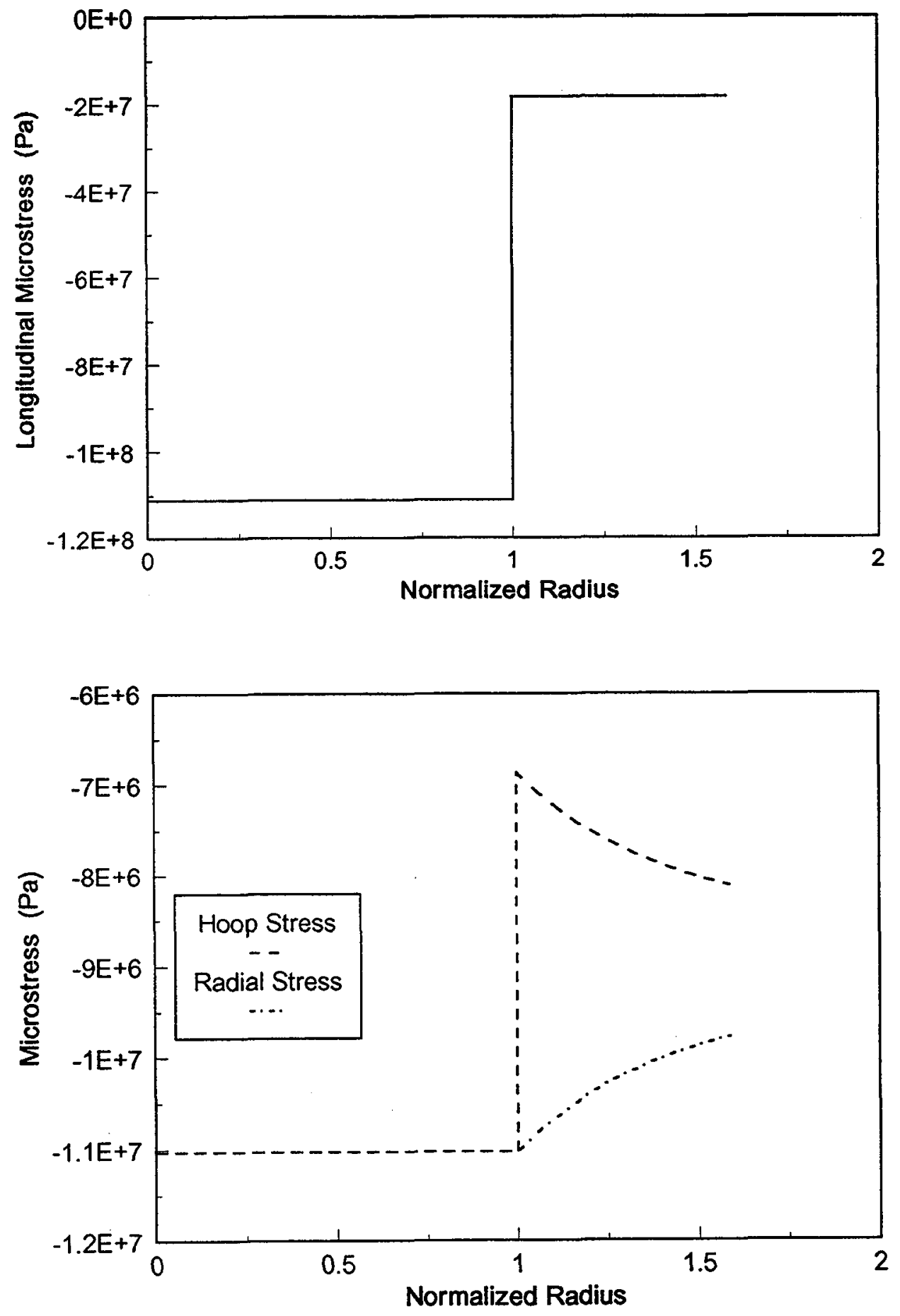

Fig. 6 Distribution of microstresses in nitinol/epoxy composite with fibers in the parent (austenite) phase $(\sigma c x=-53.6 \mathrm{MPa}, \xi=0)$. Isothermal loading cycle at 40 degs. $C$. 

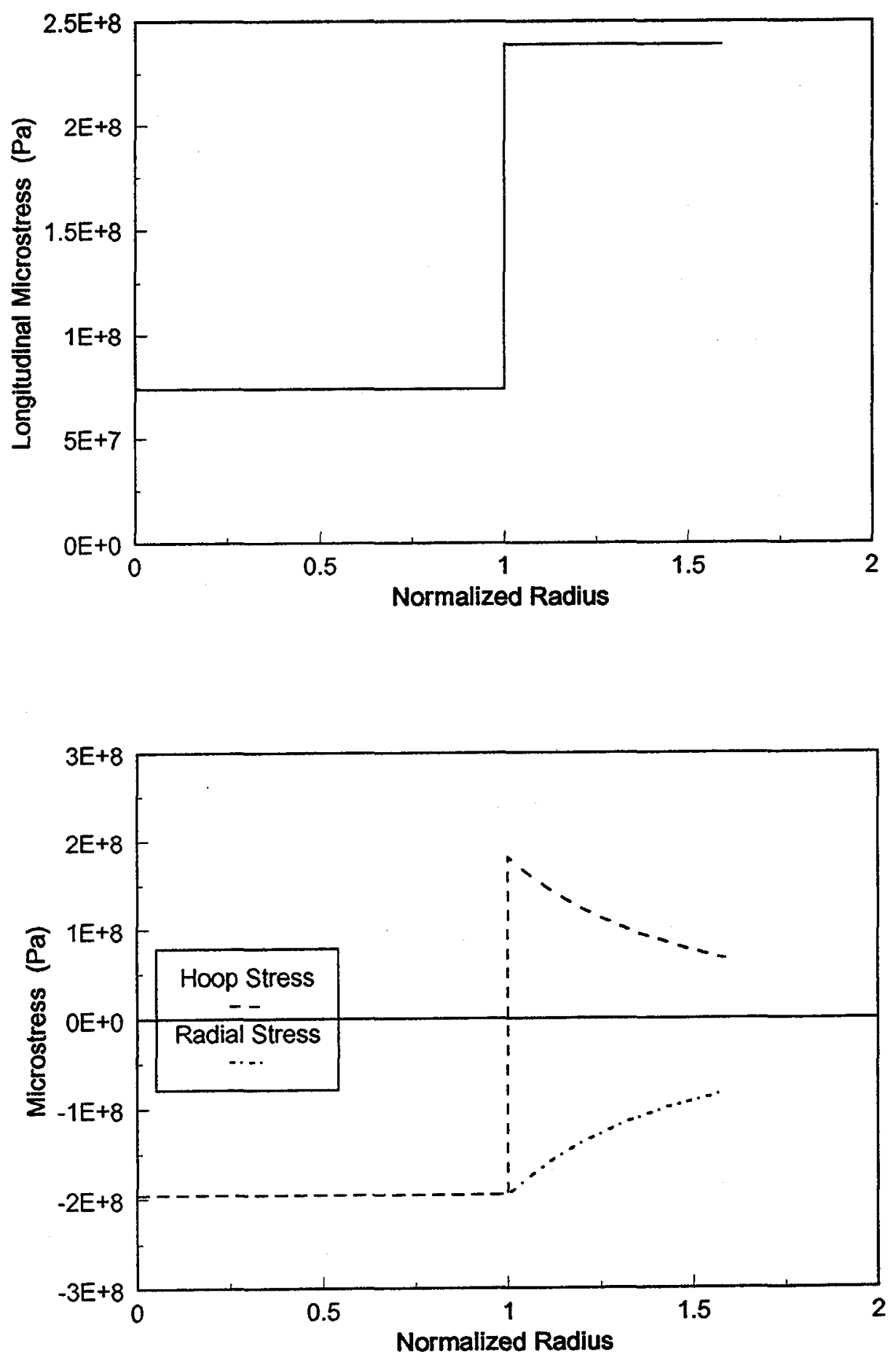

Fig. 7 Distribution of microstresses in nitinol/epoxy composite with fibers undergoing martensite phase transformation ( $\sigma c x=176 \mathrm{MPa}, \xi=0.83$ ). Isothermal loading cycle at 40 degs. C. 

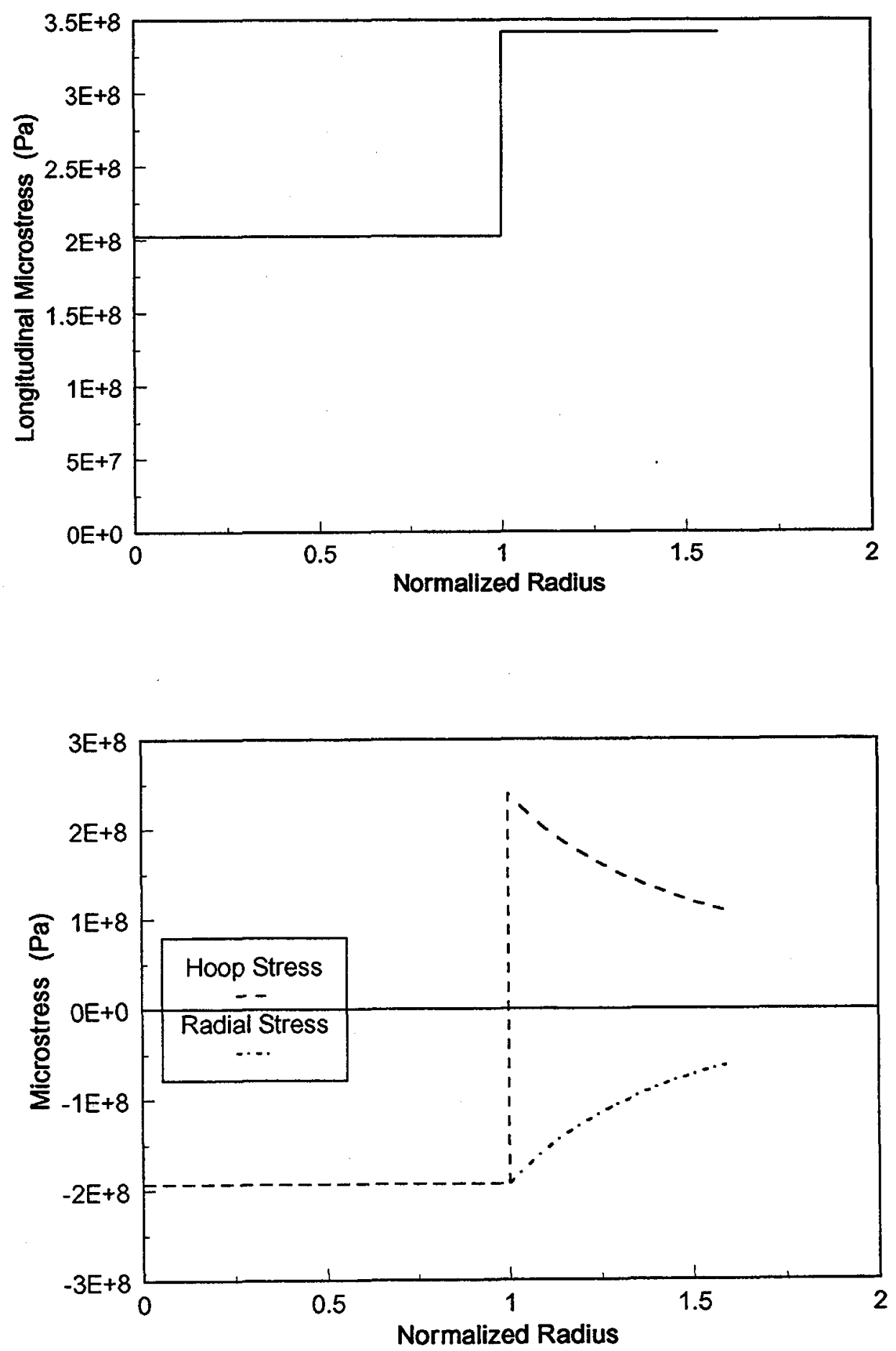

Fig. 8 Distribution of microstresses in nitinol/epoxy composite with fibers fully transformed to the martensite phase, ( $\sigma c x=288 \mathrm{MPa}, \xi=1$ ). Isothermal loading cycle at 40 degs. $C$. 

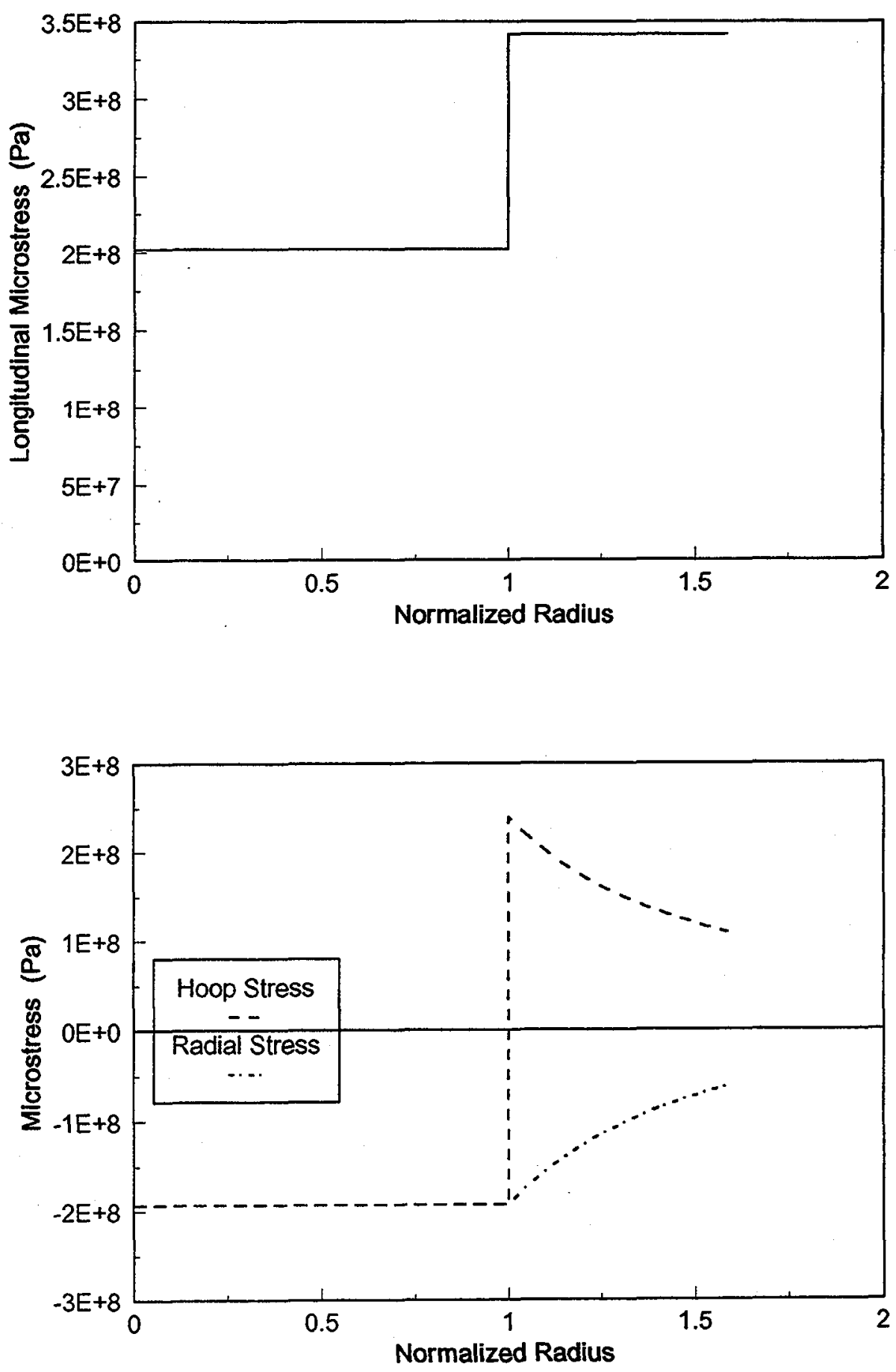

Fig. 9 Distribution of microstresses in nitinol/epoxy composite with fibers undergoing reverse transformation ( $\sigma c x=12 \mathrm{MPa}, \xi=0.40$ ). Isothermal loading cycle at 40 degs. $C$. 


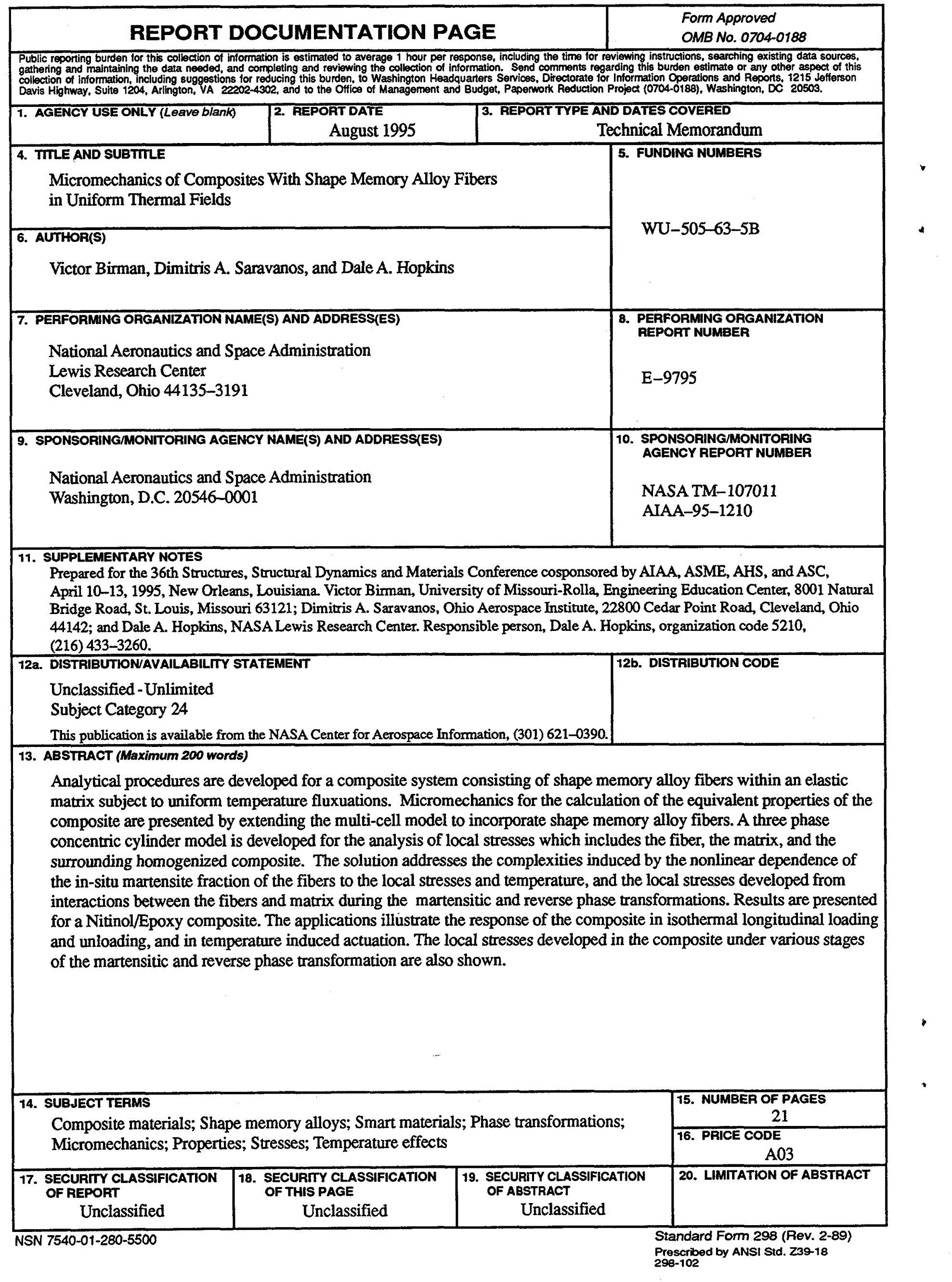


National Aeronautics and

Space Administration

Lewis Research Center

21000 Brookpark Rd.

Cleveland, OH 44135-3191

Official Business

Penalty for Private Use $\$ 300$

POSTMASTER: If Undeliverable - Do Not Return 